\title{
Numerical modelling of granular materials with spherical discrete particles and the bounded rolling friction model. Application to railway ballast.
}

\author{
Joaquín Irazábal ${ }^{\mathrm{a}}$, Fernando Salazara $^{\mathrm{a}}$, Eugenio Oñate ${ }^{\mathrm{a}, \mathrm{b}}$ \\ ${ }^{a}$ International Center for Numerical Methods in Engineering (CIMNE), Barcelona, Spain \\ ${ }^{b}$ Polytechnic University of Catalonia (UPC), Barcelona, Spain
}

\begin{abstract}
The Discrete Element Method (DEM) was found to be an effective numerical method for the calculation of engineering problems involving granular materials. However, the representation of irregular particles using the DEM is a very challenging issue, leading to different geometrical approaches. This document presents a new insight in the application of one of those simplifications known as rolling friction, which avoids excessive rotation when irregular shaped materials are simulated as spheric particles. This new approach, called the Bounded Rolling Friction model, was applied to reproduce a ballast resistance test.
\end{abstract}

Keywords: Discrete element method, bounded rolling friction model, rolling resistance torque, railway ballast, railway track stability

\section{Introduction}

Traditionally, complex geomechanic problems were addressed using refined constitutive models based on contin- 36 uum assumptions. Although these models may be accurate in the evaluation of the critical state of soils $[1,2,3,4], 37$ or the flow of bulk material masses [5], they are not able 38 to represent local discontinuities which typically play a 39 fundamental role in the behaviour of granular materials. 40 This discontinuous nature induces special features such as 41 anisotropy or local instabilities, which are difficult to un- 42 derstand or model based on the principles of continuum 43 mechanics [6].

The Discrete Element Method (DEM) is an alterna- ${ }_{45}$ tive approach that considers the granular nature of the material and provides a new insight in the constitutive model, being, nowadays, one of the most powerful and efficient tools to reproduce the behaviour of bulk materials [6]. Within the DEM approach, presented by Cundall and ${ }_{49}$ Strack [7] in 1979, each material grain is simulated as a 50 rigid particle. The deformation of the material is repre- ${ }_{51}$ sented by the interaction between the particles, allowing 52 small overlaps. The normal and tangential contact be- 53 tween the rigid particles define the material constitutive ${ }_{54}$ behaviour.

DEM has proven to be a very useful tool to obtain com- 56 plete qualitative information on calculations of groups of 57 particles [6]. However, the computational cost of contact 58 detection between Discrete Elements (DEs) is high and 59 limits the applicability of the method to some practical 60 problems, where millions of particles are typically involved. 61 This problem is especially relevant when non-spherical par- 62 ticles are employed. This limitation, together with the 63 uncertainty about the real contact mechanics and particle properties influencing the global behaviour of bulk materials [8], has led to different particle shape simplifications [9]:

- Rolling friction refers to an additional torque (rolling resistance torque) that is applied to each particle pair in contact and resists the rolling motion. This approach is typically applied to spherical DEs. Its main advantage is the low computational cost, since only the radii and the position of the centre of the spheric particles are required for the contact detection.

Contact force calculation between spherical DEs is also straightforward, as the direction of the normal force is that of the vector that joins the spheres centres.

- Sphere clusters approach consists of representing each DE particle as a group of overlapping spheres joined rigidly, thereby allowing the use of algorithms that are straightforward extensions of the efficient methods used for spheres. This approach was used to represent geomaterials $[10,11,12,13,14]$ with nonspherical particles. The total amount of spheres in the model is $n \times p$, where $n$ is the number of spheres per cluster, and $p$ is the number of particles to be considered in the model. The necessary value of $n$ to properly represent the roughness of a typical sand grain in 3D ranges from 100 to 400. In engineering calculations, where only macroscopic results are searched for, particles with $10-20$ spheres can be appropriate [15]. In both cases, there is a relevant increase of 


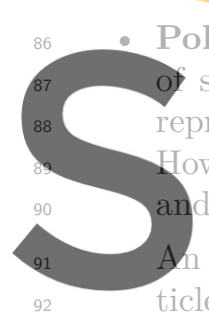
contact detection time.

It should also be noted that this approach introduces ${ }^{119}$ geometric friction due to the undesired cavities be- ${ }_{120}$ tween overlapped spheres.

Traditionally, the contact detection is split into two ${ }^{122}$ stages: Global Neighbour Search (GNS) and Local123 Contact Resolution (LCR). Although both stages can ${ }^{124}$ be optimised $[16,17,18]$, the computational time ${ }^{125}$ grows at least proportionally to the increase in the ${ }^{126}$ total amount of spheres in the model.

- Superquadrics are a family of geometric shapes de- ${ }^{128}$ fined by formulas that resemble those of ellipsoids and ${ }_{130}$ other quadrics, except that the squaring operations $s_{131}$ are replaced by arbitrary powers. Contact calculation ${ }_{132}$ between two superquadrics was addressed by different ${ }_{133}$ authors in the last ten years $[19,20,21]$.

Although superquadrics are a promising option to rep-135 resent granular materials with the DEM, the compu-136 tational cost of contact detection is high. Podlozhnyuk and Kloss [22] reported that the computational ${ }_{13}$ cost for superquadrics was 35 times higher than for spheres, in a simulation with 4860 DEs.

\section{Polyhedral particles representation allows the use} sharp edges and corner produce many kinds of g d LCR computational tin extensive effort was made
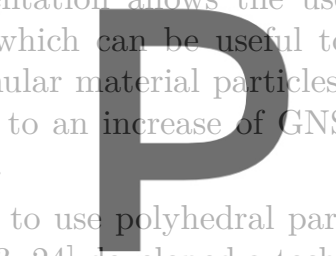
24] developed a technigue to detect contact forces between polyhedronsis

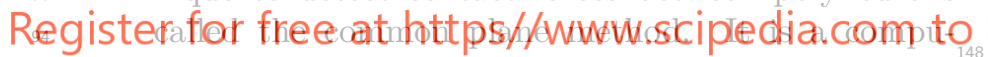
tationally expensive iterative method that replaces the contact between two polyhedrons with two plane-14s polyhedron contacts. This method was further improved by fast determination of the common plane ${ }_{151}$ [25]. Eliǎs [26] presented a new method of estimating ${ }_{152}$ the contact force between two polyhedrons based on ${ }_{153}$ calculating the intersecting volume, and applied it to ${ }_{154}$ the calculation of railway ballast behaviour. Although ${ }_{155}$ the results obtained were promising, the simulations ${ }_{156}$ involved only 120 particles, due to computational time ${ }_{157}$ issues.

Aiming to improve contact detection and force eval-159 uation, Alonso-Marroquín and Wang [27, 28] devel-160 oped the spheropolygons approach in 2D. It is based $_{161}$ in sweeping a sphere around a polygon, which leads $\mathrm{s}_{162}$ to an easier force evaluation, and a decrease in $\mathrm{LCR}_{163}$ computational time. Galindo-Torres and Pedroso [29] extended it to more complex interactions in $3 \mathrm{D}$, resulting in the spheropolyhedrons approach, 1 ich was used to predict granular materials behavio [ ].

Ahmed et al. [21] presented a new algorithm called the potential particle shapes approach. It is based in representing the particles as adjustably rounded polyhedrons. The limitation of this approach is that it is only able to represent convex particles.

In summary, the computational time of sphere cluster calculations augments proportionally to the increase of the amount of spheres in the model. For superquadrics, polyhedrons, spheropolyhedrons and potential particles, it strongly depends on the number of DEs and contacts, but the published works $[22,21,26,31]$ are limited to a few thousands of particles.

In this work, rolling friction simplification was chosen due to its simplicity and lower computational requirements.

The paper starts with the introduction of the basic formulation of the DE model used. Next, the new insight for the application of the rolling resistance torque, called the Bounded Rolling Friction (BROF) model, is presented, including some validation tests. Finally, the proposed method is used to reproduce a laboratory test that evaluates the lateral resistance of a ballast layer.

\section{Model formulation}

\subsection{Basic features \\ 2.1.1. Force evaluation}

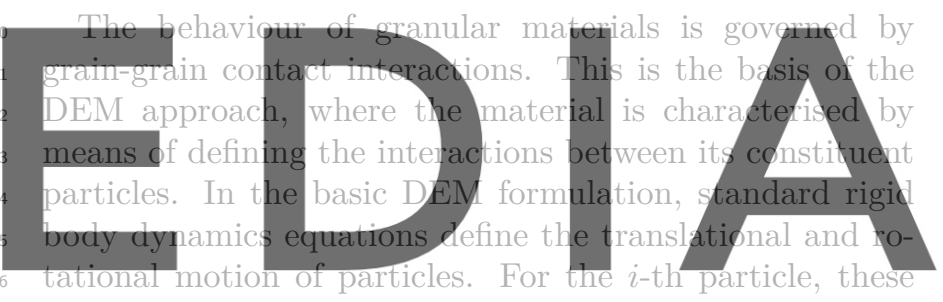

equations can be written as

download the version without the watermark

$$
\mathbb{I}_{i} \dot{\omega}_{i}=\mathbb{T}_{i}
$$

where $\ddot{\mathbf{u}}_{i}$ is the particle centroid acceleration in a fixed coordinate system $\mathbf{X}, \dot{\boldsymbol{\omega}}_{i}$ is the angular acceleration, $\mathrm{m}_{i}$ is the particle mass, $\mathbf{I}_{i}$ is the second order inertia tensor with respect to the particle centre of mass, $\mathbf{F}_{i}$ is the resultant force, and $\mathbf{T}_{i}$ is the resultant moment about the central axes.

$\mathbf{F}_{i}$ and $\mathbf{T}_{i}$ are computed as the sum of: (i) all forces and moments applied to the $i$-th particle due to external loads, $\mathbf{F}_{i}^{e x t}$ and $\mathbf{T}_{i}^{e x t}$, respectively, (ii) contact interaction forces, $\mathbf{F}^{i j}$, where $j$ is the index of the neighbouring particle ranging from 1 to the number of elements $n_{i}^{c}$ in contact with the particle under consideration $i$ and (iii) all forces, $\mathbf{F}_{i}^{\text {damp }}$, and moments, $\mathbf{T}_{i}^{\text {damp }}$, resulting from external damping.

$\mathbf{F}_{i}$ and $\mathbf{T}_{i}$ can be expressed as

$$
\begin{aligned}
& \mathbf{F}_{i}=\mathbf{F}_{i}^{e x t}+\sum_{j=1}^{n_{i}^{c}} \mathbf{F}^{i j}+\mathbf{F}_{i}^{\text {damp }} \\
& \mathbf{T}_{i}=\mathbf{T}_{i}^{e x t}+\sum_{j=1}^{n_{i}^{c}} \mathbf{r}_{c}^{i j} \times \mathbf{F}^{i j}+\mathbf{T}_{i}^{\text {damp }}
\end{aligned}
$$


where $\mathbf{n}^{i j}$ is the unit vector normal to the contact sur- ${ }^{200}$ face at the contact point.

The tangential force $\mathbf{F}_{t}^{i j}$, along the tangential direction ${ }_{210}$ $\mathbf{t}^{i j}$ (Figure 1(b)), can be written as

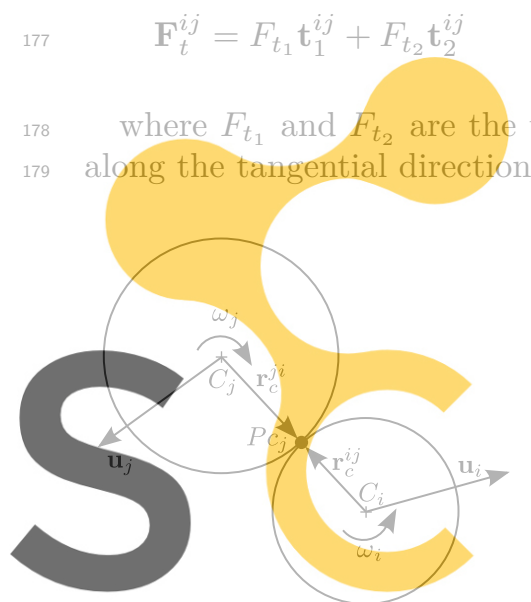

(a) Particles contact. Figure 1: Decomposition of the contact force into normal and tan- ${ }^{22}$ gential components [32]

\subsubsection{Constitutive model}

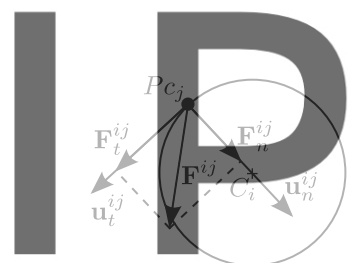

(b) Force decomposition. 215 1

\section{.}

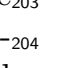
(n) 206 -

\section{, \\ 2.2. Bounded Rolling Friction (BROF) Model}

Rolling friction calculation can be addressed by different formulations. Ai et al. [36] presented four different types:

- Models type A: the direction of the rolling resistance torque is always against the relative rotation between the two contacting entities, and its magnitude depends on the material properties and the contact normal force [37].

- Models type B: the magnitude of the rolling resistance torque depends on the angular velocity [37]. There are some situations where these models do not predict rolling friction when it is required, due to its dependence on surface velocity difference between two

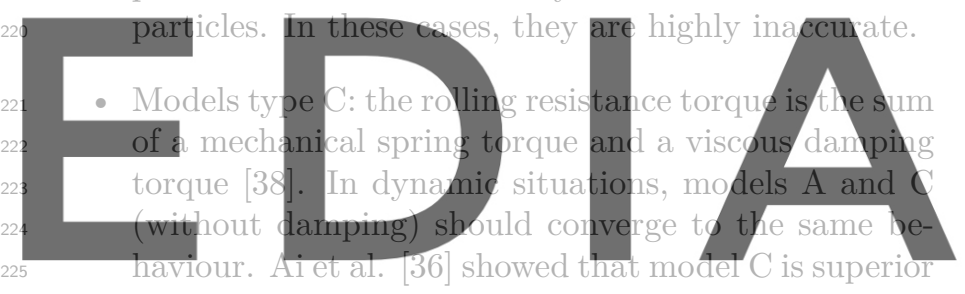

in static situations.

download the version without the watermark

- Models type D: the rolling resistance torque depends on the total rotation or rotational velocity of a particle [39]. These models are clearly inefficient [36].

Models B and D will not be further commented in this paper due to their limitations.

$\mathrm{A}$ and $\mathrm{C}$ are the most commonly used rolling friction model types [8]. In this work, model A was improved to avoid the inconsistencies appearing in static situations. The main advantage of model A over model $\mathrm{C}$ is that only one parameter is required to completely define each material rolling friction.

In model type A the rolling resistance torque $\mathbf{T}^{r}$ is given by

$$
\mathbf{T}^{r}=-e^{c}\left|\mathbf{F}^{n}\right| \frac{\boldsymbol{\omega}^{r e l}}{\left|\boldsymbol{\omega}^{r e l}\right|}
$$

where $e^{c}$ is the resistance parameter that defines the contact rolling friction, which depends on the size and material properties of the particles in contact. $\mathbf{F}^{n}$ is the normal contact force and $\boldsymbol{\omega}^{\text {rel }}$ is the relative angular velocity of the two particles in contact. Figure 2 shows schematically the implementation of the rolling friction model type A. 


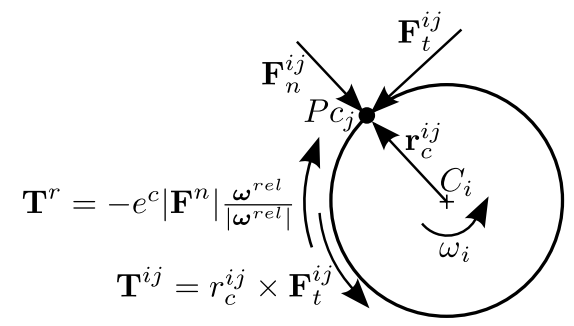

Figure 2: Scheme of rolling resistance model type A.
The material property that influences the rolling behaviour of the DE particles is called rolling friction coefficient $\left(\eta_{r}\right)$, which depends on the shape of the granular material particles: it will be higher for sharp stones than for pseudo-spherical ones. The rolling resistance parameter, $e^{c}$, depends on the rolling friction coefficient $\left(\eta_{r}\right)$ and the radius of both contacting spheres.

Till this point, $e^{c}$ was treated as the rolling resistance tricity of the contact. The need of this parameter is based284 on the fact that, when dealing with non-spherical particles285 contact, the line of action of the contact normal force does286 not pass through the centroid of the particles [8]. In the287 classical model A, the rolling resist ticle $\cdot\left(e_{i}^{c}\right)$ is considered as the pritod eefficient $\eta_{r, i}$ and the effective $r$ ista
rol
n
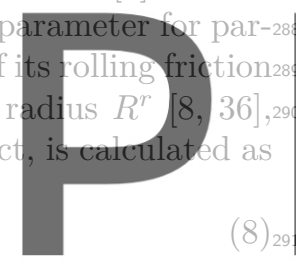
parameter. However, it can also be defined as the eccen-283

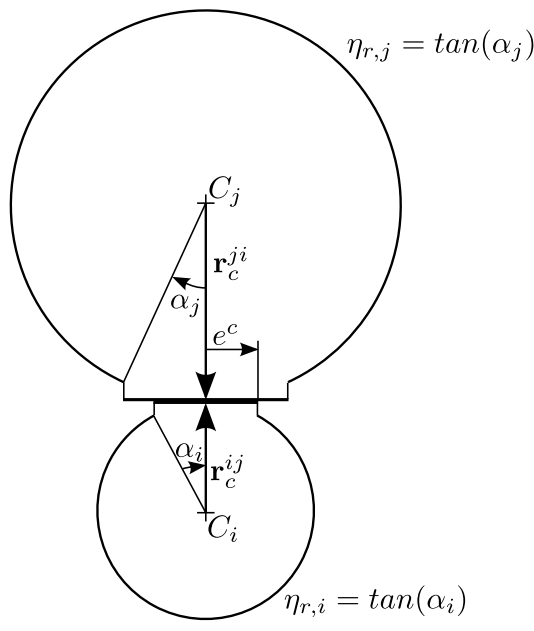

Figure 3: Schematic representation of the effect of the rolling friction parameters $e^{c}$ and $\eta_{r}$ necessary moment to stop the sphere rotation in one time step $\left(\mathbf{T}_{i}^{\max }\right)$, and not in the direction of the relative angular velocity of the two particles in contact $\left(\left|\omega^{r e l}\right|\right)$. This rithm frame-independent. was set in order to avoid discrepancies, making the algo-

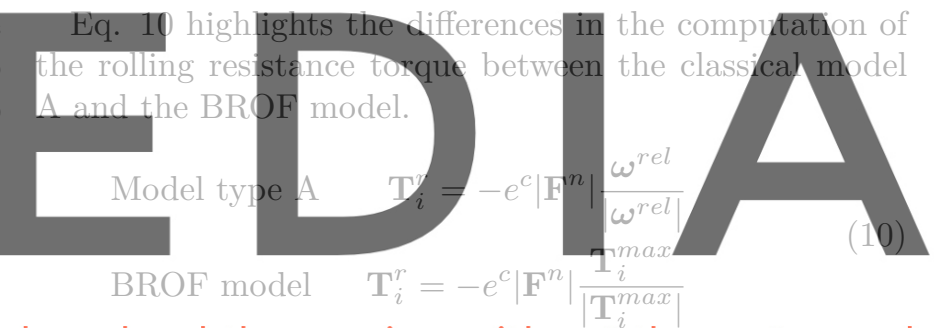
particles with very different radius sizes, because the ec-293 centricity of the contact is defined by the lowest eccentric-294 ity of the contacting particles. This feature can be clearly noticed in the scheme of Figure 3.

Ai et al. [36] outlined that model A should be used with 296 caution in static situations, because rapid oscillations in 297 the rolling resistance torque can appear due to the discon-298 tinuity in Eq. 7 at $\left|\boldsymbol{\omega}^{\text {rel }}\right|=0$. To avoid this drawback, the 299 BROF model limits the rolling resistance torque $\left(\mathbf{T}_{i}^{r}\right)$ to 300 the necessary moment to stop the sphere rotation in one 301 time step $\left(\mathbf{T}_{i}^{\max }\right)$

$$
\mathbf{T}_{i}^{\max }=\boldsymbol{\omega}_{i} \mathbf{I}_{i} \Delta t-\sum_{j=1}^{n_{i}^{c}} \mathbf{r}_{c}^{i j} \mathbf{F}^{i j}
$$$$
\text { if }\left\|\mathbf{T}_{i}^{r}\right\|<\left\|\mathbf{T}_{i}^{\max }\right\| \rightarrow \mathbf{T}_{i}^{r}=-e^{c}\left|\mathbf{F}^{n}\right| \frac{\mathbf{T}_{i}^{\max }}{\left|\mathbf{T}_{i}^{\max }\right|}
$$$$
\text { if }\left\|\mathbf{T}_{i}^{r}\right\| \geq\left\|\mathbf{T}_{i}^{\max }\right\| \rightarrow \mathbf{T}_{i}^{r}=\mathbf{T}_{i}^{\max }
$$

where $\boldsymbol{\omega}_{i}$ is the angular velocity of the sphere $i$ in theзов previous time step.

It should be noted that, within the BROF model, the 310 rolling resistance torque is applied in the direction of the 311 06

\section{s.}

\author{
309
}

\section{the}

cownload the version without the watermark Anitescu [40], avoids undesirable oscillations in the spheres spin.

\subsection{Software}

The data structures and algorithms have all been implemented through the Kratos multiphysics software suite [41], an Open-Source framework for the development of numerical methods for solving multidisciplinary engineering problems. Within Kratos multiphysics, a DEM code called DEMPack (www.cimne.com/dempack/) was implemented.

\section{BROF model validation}

Two of the benchmark cases described by Ai et al. [36] were selected for the validation of the BROF model. In both cases, the same material properties and simulation parameters described in [36] were used.

\subsection{Test case 1: sphere with initial velocity rotating over} a flat surface [36]

The first test adopted is a single sphere (with rolling iction) rotating over a flat surface. To develop the simuation, a sphere is placed over a rigid surface letting it move 
The material properties and simulation parameters used in test cases 1 and 2 are summarised in Table 1 .

by its own weight until it achieves equilibrium. Then, an initial translational velocity $\left(v_{0}=1.0 \mathrm{~m} / \mathrm{s}\right)$ is applied to the sphere. The test case layout is shown in Figure 4.

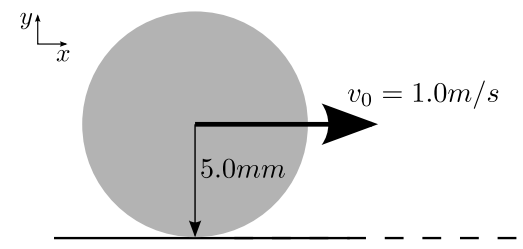

Figure 4: Initial layout of test case 1
Table 1: Material properties and calculation parameters used in test

cases 1 and 2 .

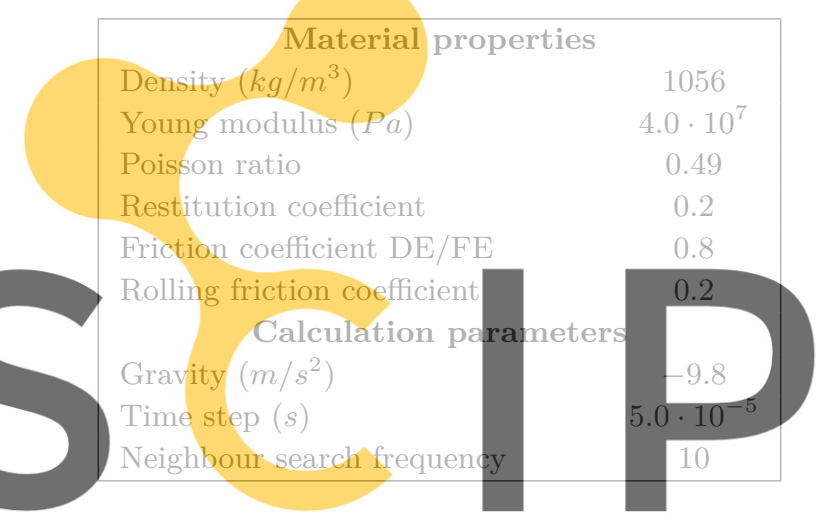

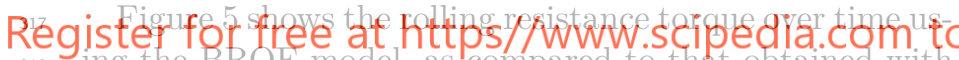
ing the BROF model, as compared to that obtained with simulation, the rolling resistance torque in both models is a constant value given by Eq. 7. However, once the sphere reaches its final position, differences between both models arise. In the classic model $\mathrm{A}$, the torque oscillates between a positive and a negative value with the same magnitude. ${ }_{345}$ The BROF model overcomes this inconvenience thanks to $_{346}$ the limitation imposed in eq. 9, and leads to an equilib- 347 rium situation where the rolling resistance torque and the particle angular velocity are zero.

The torque instability for model A generates oscillations ${ }^{348}$ in angular velocity, which are also eliminated with the ${ }^{349}$ BROF model. Although their magnitude is low for the 350 test case 1, the kinetic energy generated can be relevant ${ }_{351}$ in simulations involving a large amount of particles.

With model C, damping is necessary to avoid oscillations353 in a static situation. Without damping, the behaviour 354 would be similar to the behaviour of model A, but the os-355 cillating frequency does not depend on the step: it depends356 on the rolling stiffness and the mass of the sphere. 357

The graph in Figure 6 shows the response of the $\mathrm{BROF}_{358}$ model and the classic rolling friction model $\mathrm{C}$ with a damp-359 ing ratio $\delta_{r}=0.3$. It can be appreciated that, in model $\mathrm{C}, 360$

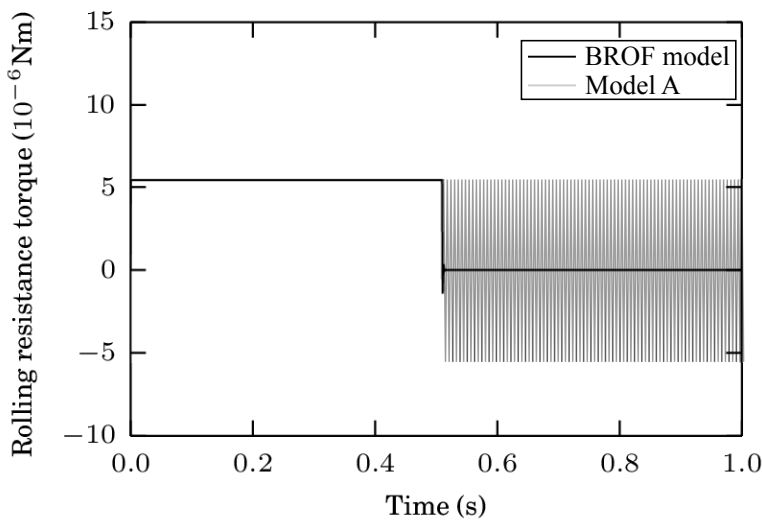

Figure 5: Comparison between rolling resistance torque obtained applying the classic rolling friction model A and the BROF model. some oscillations still appear although damping is applied. The amplitude of the oscillation decreases gradually with time.

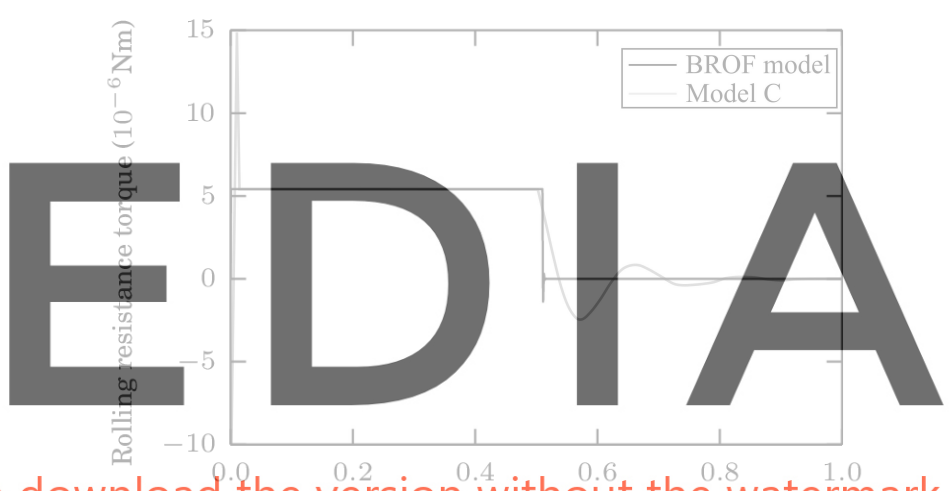
download the version without the watermark

Figure 6: Comparison between rolling resistance torque obtained applying the classic rolling friction model $\mathrm{C}$ with a damping ratio $\delta_{r}=0.3$ and the BROF model.

The results obtained for test case 1 show that BROF model outperforms models $\mathrm{A}$ and $\mathrm{C}$. The difference is less relevant for model C.

\subsection{Test case 2: sphere with initial angular velocity rotat- ing over an inclined surface [36]}

The aim of the second test case is to evaluate the influence of varying the rolling friction coefficient in the BROF model. It consists of a sphere rolling up a slope with an angle of $\beta=10$ degrees, as shown in Figure 7 . The sphere has the same properties as in test case 1 (see Table 1). In this case the sphere is positioned over the rigid surface allowing it to move by its own weight, but restringing its movement in the $\mathrm{x}$ direction (see Figure 7 ). When the sphere come to rest, $\mathrm{x}$ movement restriction is removed and an initial translational velocity $v_{0}=1.0 \mathrm{~m} / \mathrm{s}$, parallel to the slope, is applied. 


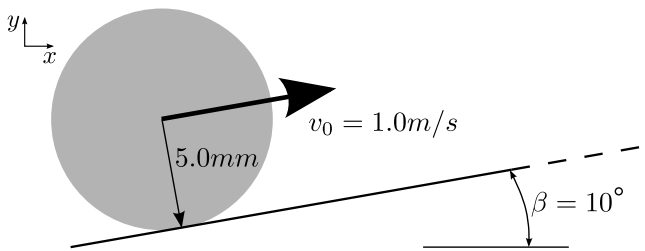

Figure 7: Initial layout of test case 2.
In order to evaluate the influence of the rolling friction ${ }^{384}$ coefficient in the sphere response, two other values of the ${ }^{385}$ rolling friction coefficient $\eta_{r}$ were considered. When $\eta_{r}$ is lower than 0.176 (which corresponds to a rolling friction angle $\alpha=10$ degrees) the sphere should roll back downwards after reaching its highest point. When $\eta_{r}$ is sufficiently large (more than 0.176), the sphere should be stopped by a resistance torque that prevents the downward rolling due to gravity.

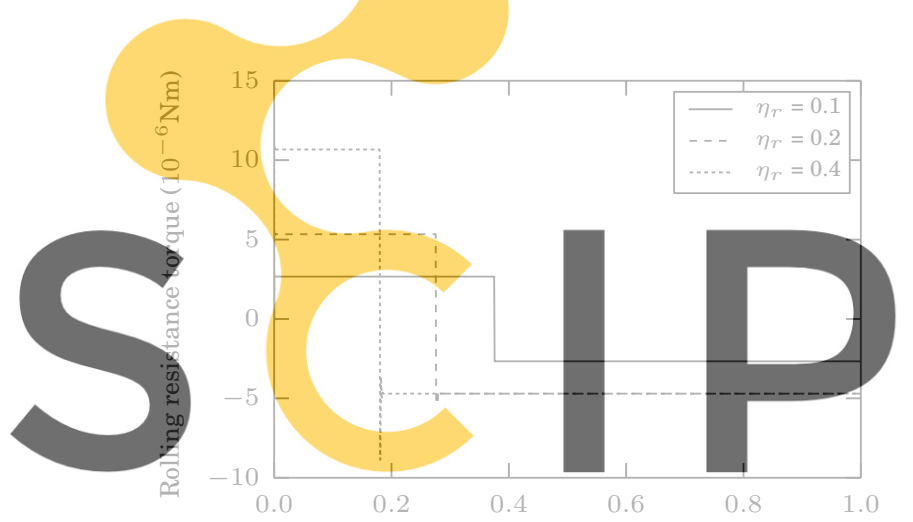

Register for free at https T/We(s) W.scipedi
(a) Rolling resistance torque versus time.

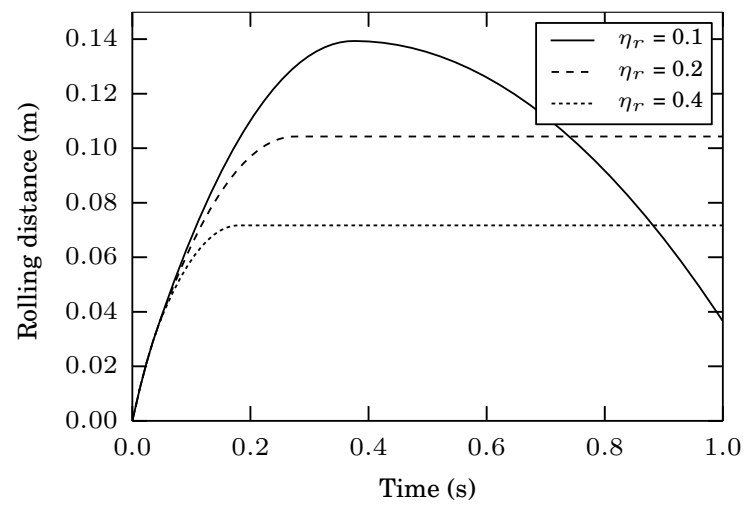

(b) Rolling distance versus time.
Figure 8: Test case 2 results for three different rolling friction coef-390 ficients $\eta_{r}=0.1,0.2$ and 0.4 applying the BROF model.
Figure 8(a) shows the evolution of the rolling resistance torque over time. It is worth noting that applying the ${ }^{392}$ BROF model, the rolling resistance torque in dynamic sit-393 uations is constant for a specific value of the rolling friction 394 coefficient. However, when the particle comes to rest, the rolling resistance torque is set to a specific value, which is the necessary torque to stop the sphere rotation in one time step. This feature can be clearly appreciated in Figure 8(a), for $\eta_{r}=0.2$ and 0.4 , where the rolling resistance torque is the same independently of the value of the rolling friction coefficient.

Figure 8(b) shows the sphere rolling distance over time. It can be observed that, with a higher rolling friction coefficient, the sphere spin stops faster. As expected, the sphere rolls back downwards after reaching its highest point for $\eta_{r}=0.1$.

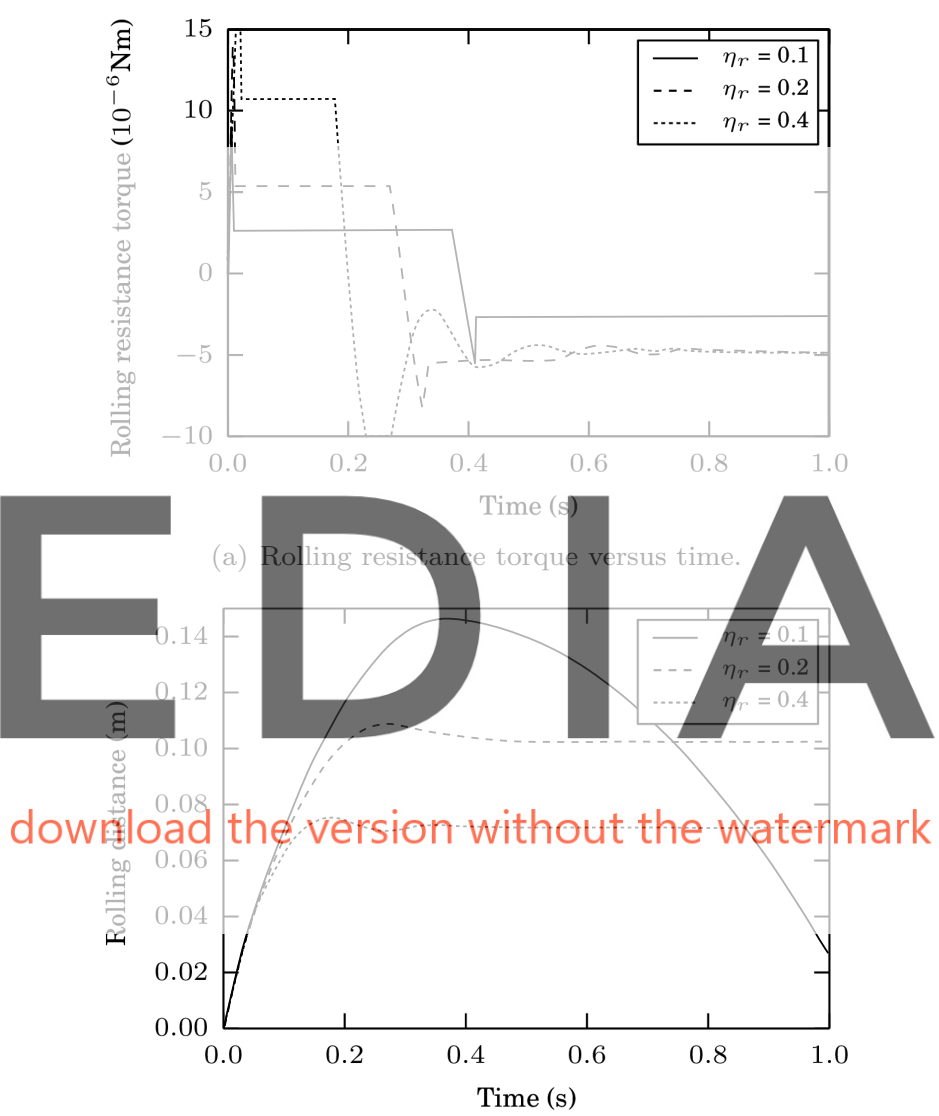

(b) Rolling distance versus time.

Figure 9: Test case 2 results for three different rolling friction coefficients $\eta_{r}=0.1,0.2$ and 0.4 applying the classic rolling friction model $\mathrm{C}$ with a damping ratio $\delta_{r}=0.3[36]$.

Figure 9 presents the results obtained by Ai et al. [36] with the classic rolling friction model $\mathrm{C}$ with a damping ratio $\delta_{r}=0.3$. Although the rolling resistance torque is similar, BROF model avoids oscillations with only one parameter to calibrate.

\section{Railway ballast behaviour calculation}

\subsection{Ballast characterisation}

Railway ballast refers to the layer of crushed stones placed between and underneath the sleepers. The purpose 
of this layer of granular material is to provide drainage 447 and structural support for the dynamic loading applied by 448 trains [42].

The ballast layer is relatively inexpensive and easy to450 maintain. However, the demands over the ballasted track ${ }_{451}$ are increasing due to the faster, heavier and more frequent 452 trains, which yields to the necessity of a better understand-453 ing of its mechanics and the way in which it resists lateral 454 and vertical loads [21].

Mechanical testing on specimens of railway ballast is difficult to carry out in traditional laboratory devices owing to the large particle size [43]. Thus, there is interest in developing simulation techniques that enable the numerical analysis of the mechanical behaviour of ballast. Railway ballast is an ideal material to be calculated with the DEM [21], due to its granular nature and relatively large grain size, compared with the depth of the ballast layer.

Some material properties of ballast are well documented in technical literature. In this work, the following values were adopted:

\section{Density: $2700 \mathrm{~kg} / \mathrm{m}^{3}[44]$.}

- Particle size: ballast granulometry is regulated [45]. Following the indications of European standards, the

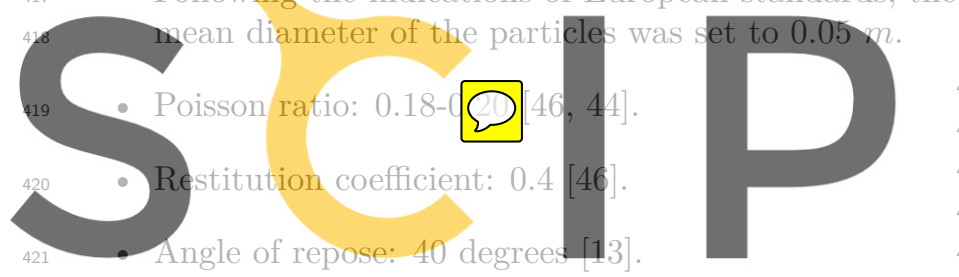

\subsection{Angle of repose}

The angle of repose is defined as the slope of a pile of granular material laid up on the ground without any other support [50]. The importance of this material property is that it controls all parameters that affect the behaviour of large amounts of granular material (friction between particles, shape and size of different grains), allowing their evaluation in a simple way.

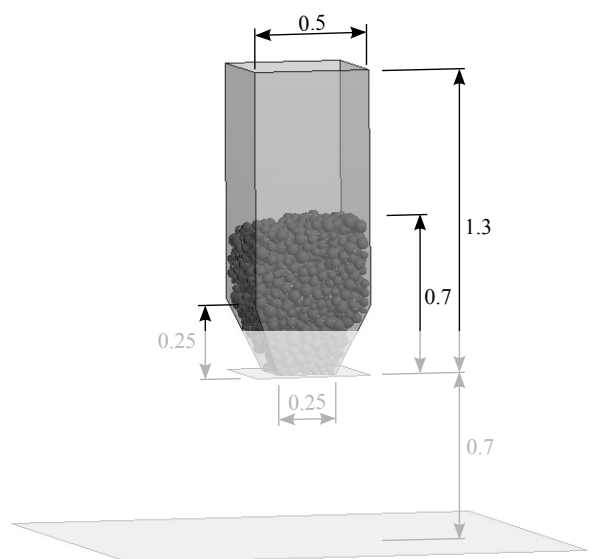

Figure 10: Simulation layout (measurements in meters) [13].

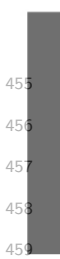

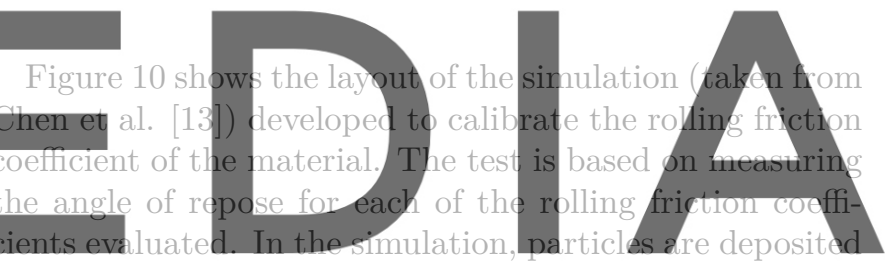
from a hopper with a squared aperture of $25 \mathrm{~cm}$ side, lo-

thors suggest $E=30$ GPa $[47,48]$. However, contacts be-463 tween real ballast stones are not Hertzian, as the particies464 have rough and non-spherical surfaces [49]. For rough sur-465 faces, the contact radius of curvature is much smaller than ${ }_{466}$ for idealised spherical shapes. As a consequence, the appropriate value of the Young modulus when using spheres is lower. Ahmed et al. [21] used values of shear modulus $(G)$ between 1 and $10 G P a$, that corresponds to a value of the Young modulus between 2.36 and 23.6 GPa for the chosen Poisson ratio $(\nu=0.18)$. In this work, we tested four values within that range: $E=5.9,11.8,17.7$ and 23.6 GPa, which correspon $G=2.5,5,7.5$ and 10 GPa.

The friction coefficiermetween ballast stones depends on the time and the load cycles suffered by ballast stones. According to Melis [44], the friction angle should always be between 30 and 40 degrees (friction coefficient between 0.577 and 0.839 ). In this work, a value of 0.6 was selected, following Chen et al. [13].

As mentioned before, ballast particles were represented as spherical DEs with rolling friction. The value of the 467 rolling friction coefficient was calibrated to reproduce the 468 angle of repose of ballast, as described in the following ${ }_{469}$ section.

\section{doweflogat etherversion without the watermark} 2. The critical time step of the system is determined by its highest natural frequency, and it depends on the mass and the stiffness of the particles. For that reason, different time steps were used for each simulation.

Table 2: Data summary.

\begin{tabular}{|lc|}
\hline \multicolumn{2}{|c|}{ Material properties } \\
Density $\left(\mathrm{kg} / \mathrm{m}^{3}\right)$ & 2700 \\
Poisson coefficient & 0.18 \\
Young modulus $(\mathrm{GPa})$ & $5.9 / 11.8 / 17.7 / 23.6$ \\
Friction coefficient & 0.6 \\
Restitution coefficient & 0.4 \\
Rolling friction coefficient & $0.2 / 0.25 / 0.3$ \\
& \\
Time step $(\mu \mathrm{s})$ & $8.0 / 6.0 / 5.0 / 4.0$ \\
Neighbour search frequency & 10 \\
\hline
\end{tabular}

Figure 11 shows the angle of repose obtained for each value of the rolling friction coefficient. It corresponds to the tests for $E=17.7 \mathrm{GPa}$, though the results were independent of the Young modulus (results not shown). 


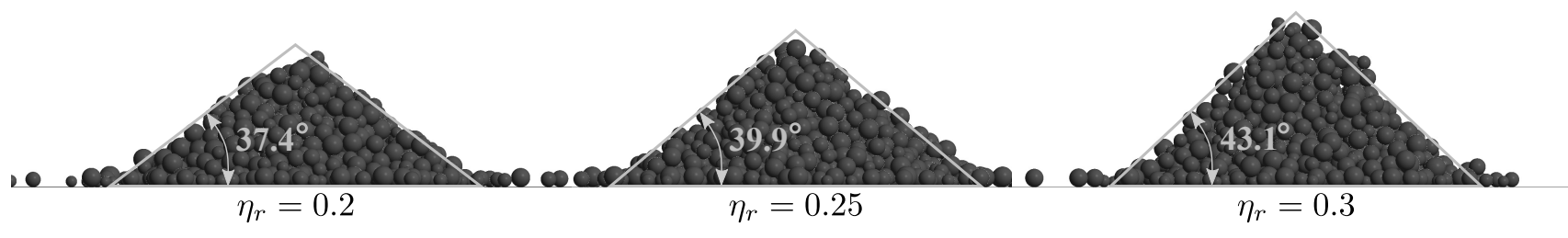

Figure 11: Repose angle of the granular material for different rolling friction parameters $(E=17.7 \mathrm{GPa})$.

Since the angle of repose of ballast is 40 degrees, the rolling friction coefficient was set to 0.25 for the benchmark test described in the following section.

It should be noted that the rolling friction approach can be useful to reproduce other granular materials with spherical DEs.

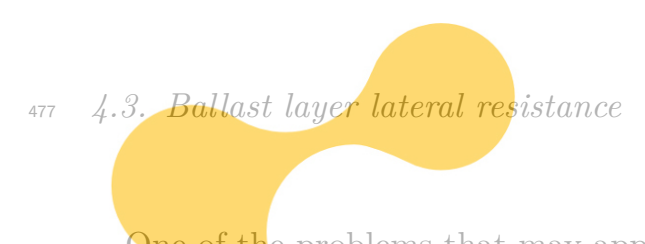

One of the problems that may appear in railway infrastructures is lateral buckling, which is one of the most criticaltroubles in railroad tracks 5 1]. It can greatly affect the circulation and may cause catastrophic Lateral buckling can be caused loads, being relatively common viations in temperature between this yeason, lateral resistance of 1 most important parameters regarding track stability. In 487 this context, the ballast plays a crucial role [51].

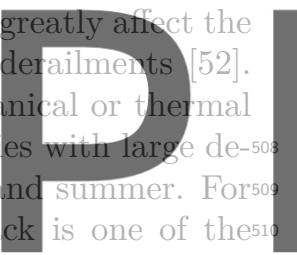
Register for free at https//www.scipedia.com to

oped a numerical simulation to evaluate the lateral resis- ${ }^{513}$ tance force of a ballast layer against a sleeper with imposed ${ }^{51}$ motion.

A reference experimental test [53] was reproduced numerically, and the results were compared.

$$
494
$$

\subsubsection{Reference test}

Zand and Moraal [53] conducted a series of threedimensional ballast resistance tests using a rail track panel. Those tests were performed in the Roads and Railways Research Laboratory of the Delft University of Technology (TU Delft).

The tests consisted of a track panel with five sleepers inside a ballast bed (Figure 12). Lateral load was applied by means of two diagonal rods connecting the hydraulic actu-517 ator $(150 \mathrm{kN})$ to the track section. Two connecting beams518 were welded between the rails to reinforce the track panel ${ }_{519}$ enabling a more uniform load application. The motion of $f_{52}$ the track panel was imposed and the opposing force was521 measured.
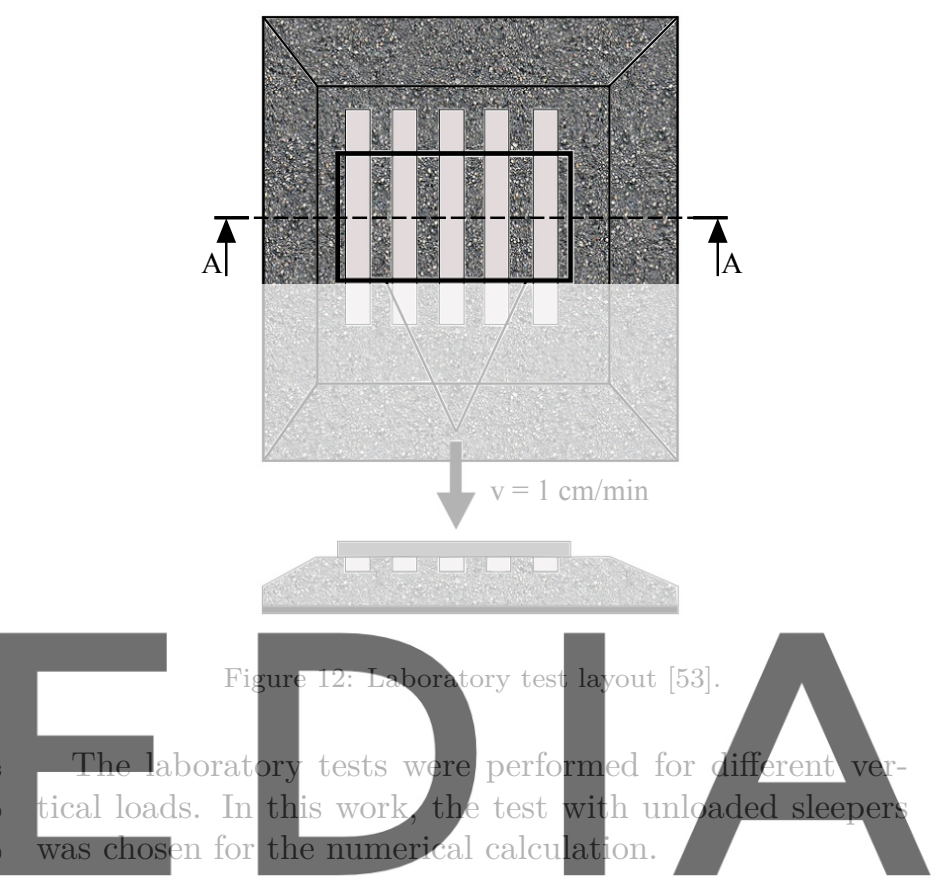

4.3.2. DE model the laboratory test, but for only one sleeper, instead of five (see Figure 13). Lateral resistance test simulations were developed using spherical discrete elements with rolling friction.

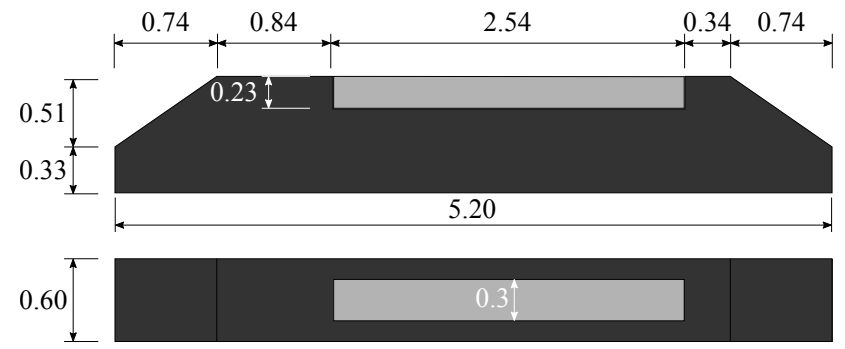

Figure 13: Test geometry for calculating ballast lateral resistance force against sleeper movement (distances in meters).

Particles initial distribution is a key parameter that has not been already mentioned, since it is specific for numerical modelling, though irrelevant for the case described in section 4.3.1.

To start the calculation, the volume has to be filled with spherical DEs. Although there exist sphere meshers (e.g. 


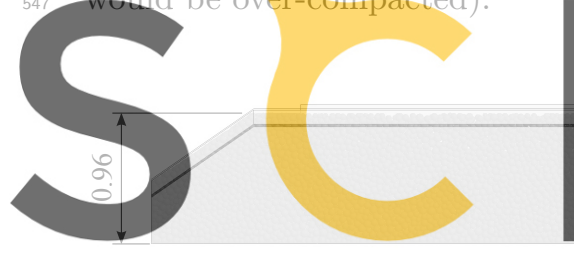

GiD pre and post-processor sphere mesher, http://www. 552 gidhome.com/), the result do not always meet the desired553 material compactness. As a result, new alternatives need554 to be considered to address the problem.

Tran [54] proposed the so-called gravitational packing556 technique to generate DE samples for granular material557 simulations. It consists in assigning the particles a zero558 friction coefficient value, and letting them to freely fill the559 volume under consideration. This leads to a high particles60 compactness, though requires a pre-simulation. This is the561 method applied in this work.

In this specific case, an auxiliary surface is needed to maintain the slope of the the embankment when the ma-563 terial friction angle is zero.

Figure 14 shows the layout of the numerical model at the beginning and at the end (time $=2.5 \mathrm{~s}$ ) of the pre- ${ }_{566}$ simulation. The auxiliary surfaces move downwards together with the granular material in order to maintain the desired geometry. In Figure 14 it can also be seen that an auxiliary sleeper, higher than the real one, was used to keep the geometry of the ballast layer.

At the end of the pre-simulation, it was verified that the value of the vertical force on the upper part of the auxiliary surfaces was zero (otherwise, the ballast layer would be over-compacted).
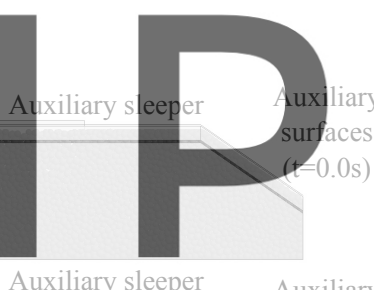

Auxiliary

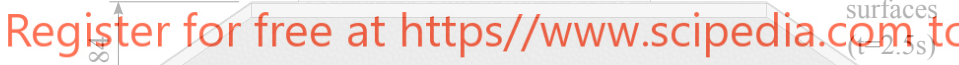
$\stackrel{\infty}{\circ}$

Figure 14: Auxiliary surfaces used to keep the geometry during the ${ }^{567}$ pre-simulation.

The particle arrangement at the end of the pre- -570 simulation was the starting point of the laboratory test ${ }^{571}$ numerical calculation. The DE mesh, consisting of $21,708^{572}$ spheres, is shown in Figure 15.

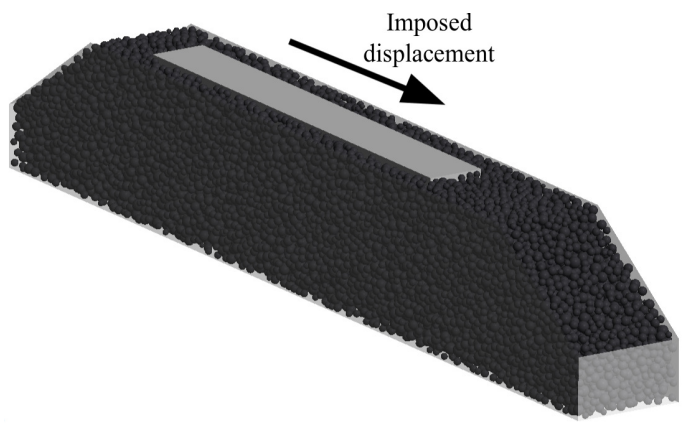

Figure 15: Initial configuration for the ballast resistance numerical ${ }^{586}$ test.
The friction between ballast and the outer walls was considered null to simulate a continuous domain with mirrored particles. Hence, the results of the numerical model can be compared to those obtained in the experiment, where the lateral force was applied to 5 sleepers.

The material properties and calculation parameters were defined in Table 2. The rolling friction coefficient was set to 0.25 , based on the results of section 4.2 . The value of the friction coefficient between the ballast stones and the sleeper was taken from the reference study [53], where it was computed experimentally.

\subsubsection{Results}

Figure 16 shows the results of the lateral resistance force versus the sleeper displacement. The numerical and the experimental results are compared.

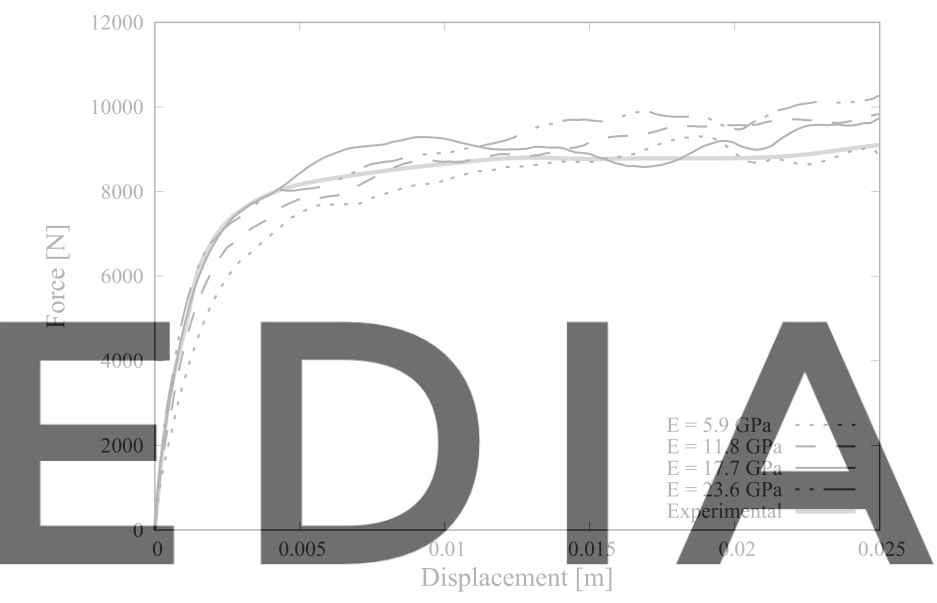

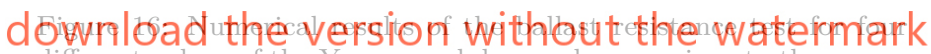
different values of the Young modulus, and comparison to the experimental test.

It can be observed that the results in the first loading stages for $E=17.7 G P a$ and $E=23.6 G P a$ are almost identical, and close to the experimental curve. For lower values of $E$, the slope is also lower. The differences in terms of the maximum resistance force are less relevant, with certain erratic behaviour.

These results suggest that for this test, the influence of $\mathrm{E}$ is negligible provided that some value greater than 17.7 $G P a$ is chosen. Since lower values allow for larger time steps and low computational time, it is advantageous to use $E=17.7 G P a$.

An interesting feature of the numerical methods is that they allow obtaining results difficult to measure in experimental facilities. As an example, the percentage of the lateral resistance force exerted by ballast against each face of the sleeper can be computed. This information can be useful to optimise the geometry of the cross-section to increase the lateral resistance force under different situations.

Figure 17 shows the results. It can be seen that at the start of the simulation, $50 \%$ of the resisting force is due to 


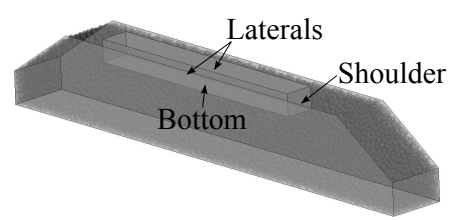

(a) Sleeper faces.

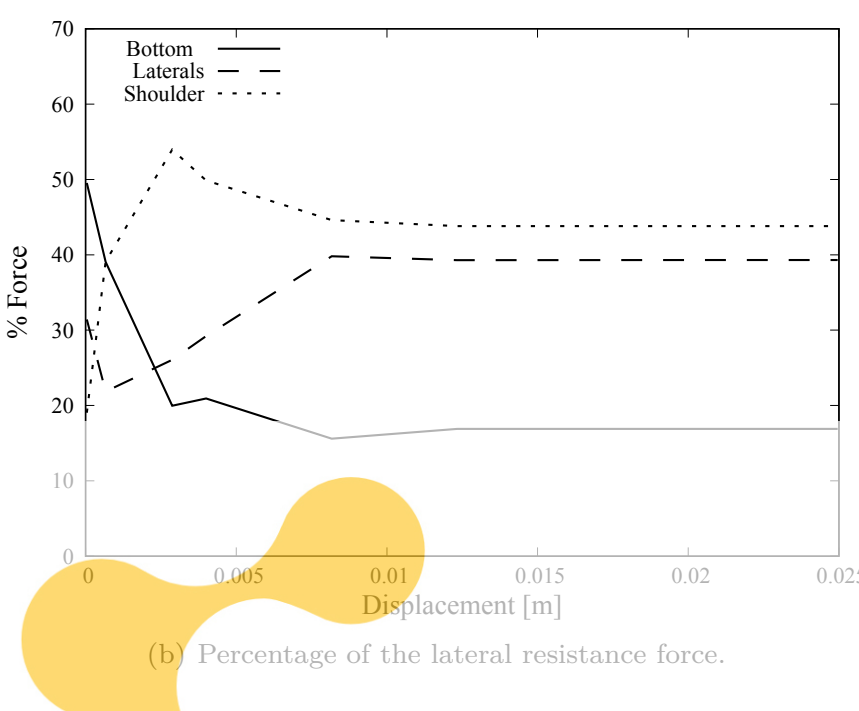

Figure 17: Percentage of the lateral resistance force acting on each ${ }^{63}$

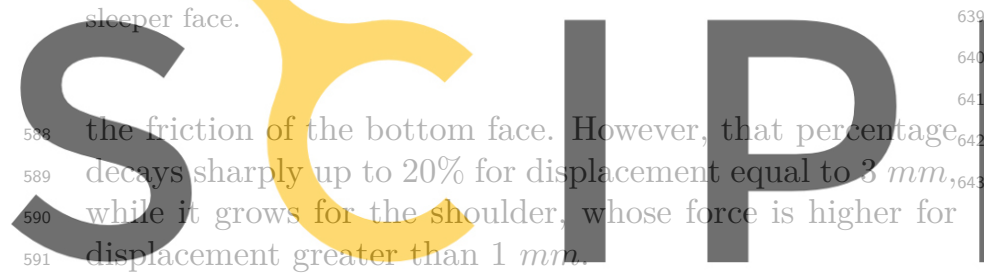

According to these results, the most effective way to ${ }^{644}$

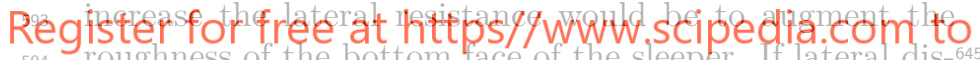$$
636
$$

637
To calibrate the BROF model $\eta_{r}$ parameter, the angle of repose of the granular material can be used, since it is easy to obtain it in the laboratory. In the case study presented, an angle of repose of 40 degrees was obtained for ballast with $\eta_{r}=0.25$.

The BROF model with spherical DEs was used to reproduce an experimental test on the lateral resistance of ballast against a sleeper with imposed motion. The initial stiffness was correctly reproduced, and the maximum force was captured with an error of almost the $6 \%$.

DEM allows detailed analyses of the system response, which are often difficult to carry out in laboratory. In the benchmark presented, the evolution of the relative influence in the resistant force of each component of the ballast layer was identified.

The results showed some degree of dependence on the Young modulus value. In particular, they suggest that a minimum value of $17.7 G P a$ (correspondent to a shear modulus of $7.5 \mathrm{GPa}$ ) should be considered. Hence, calibration of this parameter seems advisable before applying this model to reproduce ballast behavio $\bigcirc$ der different load conditions.

Although the results suggest that spherical DEs appropriate to reproduce the macroscopical behav $Q$ f

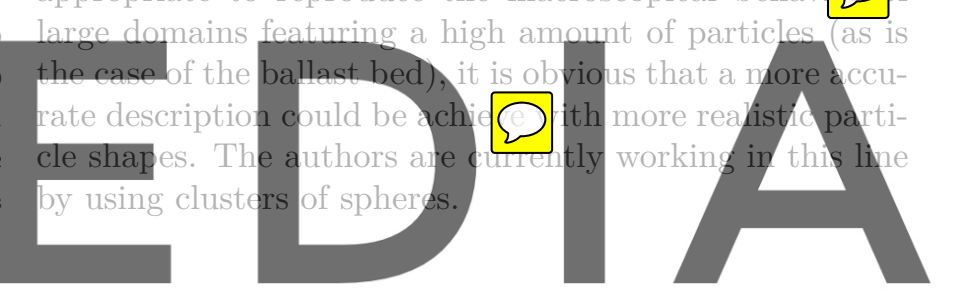
Acknowledgement $\Omega$ placements greater than $1 \mathrm{~mm}$ were allowed, the geometry ${ }^{646}$ of the shoulder should be optimised.

A more comprehensive analysis would be required to ${ }^{648}$ draw conclusions in a practical case, including the analysis ${ }^{649}$ of loaded scenarios.

\section{Summary and conclusions}

A new model, called the Bounded Rolling Friction ${ }_{656}^{655}$ (BROF), for the computation of rolling friction for spher- ${ }_{657}^{656}$ ical DE particles was presented. Besides providing similar658 results than the previous rolling friction models in dynamic ${ }^{659}$ situations, it includes a limitation to the angular velocity ${ }_{661}^{660}$ in order to avoid undesirable sphere rotation when the par- ${ }_{662}$ ticle is almost at rest. The BROF model was compared 663 with previous rolling friction models, concluding that the ${ }^{664}$ results are accurate, with only one parameter $\left(\eta_{r}\right)$ to be $\mathrm{e}_{666}^{665}$ calibrated. BROF model sensitivity to changes in $\eta_{r}$ was $_{667}$ also checked.

It can be concluded that the BROF model outperforms ${ }_{670}^{609}$ previous approaches for modelling irregular particle shapes ${ }_{671}^{670}$ with spherical DEs.

\section{download the versilon without the watermark} port Spanish MINECO within the BALAMED (BIA201239172 ) and MONICAB (BIA2015-67263-R) projects.

We appreciate the reviewers the time taken to provide valuable comments that have improved undoubtedly the quality of the article.

[1] J. Duncan, State of the Art: Limit Equilibrium and FiniteElement Analysis of Slopes, J Geotech Eng-ASCE 122 (7) (1996) 577-596.

[2] Y. Dafalias, M. Manzari, A Critical State Two-surface Plasticity Model for Sands, Géotechnique 47 (2) (1997) 255-272.

[3] B. Indraratna, S. Nimbalkar, M. Coop, S. W. Sloan, A constitutive model for coal-fouled ballast capturing the effects of particle degradation, Comput Geotech 61 (2014) 96-107.

[4] M. Esmaeili, A. Khodaverdian, H. K. Neyestanaki, S. Nazari, Investigating the effect of nailed sleepers on increasing the lateral resistance of ballasted track, Comput Geotech 71 (2016) $1-11$.

[5] F. Salazar, J. Irazábal, A. Larese, E. Oñate, Numerical modelling of landslide-generated waves with the particle finite element method (PFEM) and a non-Newtonian flow model, Int J Numer Anal Met 40 (6) (2015) 809-826.

[6] N. Belheine, J. Plassiard, F. Donzé, F. Darve, A. Seridi, Numerical Simulation of Drained Triaxial Test using 3D Discrete Element Modeling, Comput Geotech 36 (1-2) (2009) 320-331.

[7] P. Cundall, O. Strack, A Discrete Numerical Model for Granular Assemblies, Géotechnique 29 (1) (1979) 47-65. 
[8] C. M. Wensrich, A. Katterfeld, Rolling friction as a technique744 for modelling particle shape in DEM, Powder Technol 217745 (2012) 409-417.

[9] J. E. Lane, P. T. Metzger, R. A. Wilkinson, A Review of Dis-747 crete Element Method (DEM) Particle Shapes and Size Distri-748 butions for Lunar Soil, Tech. rep., NASA (2010).

[10] T. Matsushima, J. Katagiri, K. Uesugi, A. Tsuchiyama,750 T. Nakano, 3D Shape Characterization and Image-Based DEM751 Simulation of the Lunar Soil Simulant FJS-1, J Aerospace Eng752 $22: 1$ (15) (2009) 15-23.

[11] X. Garcia, J. Xiang, J.-P. Latham, J.-P. Harrison, A clustered754 overlapping sphere algorithm to represent real particles in dis-755 crete element modelling, Géotechnique 59 (9) (2009) 779-784. 756

[12] J.-F. Ferellec, G. R. McDowell, A method to model realistic757 particle shape and inertia in DEM, Granul Matter 12 (5) (2010)758 459-467.

[13] C. Chen, G. R. McDowell, N. H. Thom, Investigating geogrid-760 reinforced ballast: Experimental pull-out tests and discrete el-761 ement modelling, Soils Found 54 (1) (2014) 1-11.

[14] N. T. Ngo, B. Indraratna, C. Rujikiatkamjorn, DEM simulation763 of the behaviour of geogrid stabilised ballast fouled with coal,764 Comput Geotech 55 (2014) 224-231.

$15]$ B. Indraratna, N. T. Ngo, C. Rujikiatkamjorn, J. S. Vinod, Be-766 havior of Fresh and Fouled Railway Ballast Subjected to Direct767 Shear Testing: Discrete Element Simulation, Int J Geomech768 14 (1) (2014) 34-44.

[16] K. Han, Y. Feng, D. Owen, Performance comparisons of tree-770 based and cell-based contact detection algorithms, Eng Com-771 putation 24 (2) (2007) 165-181.

[17] D. A. Horner, J. F. Peters, A. Carrillo, Large Scale Discrete El-773 ement Modeling of Vehicle-Soil Interaction, J Eng Mech-ASCE774 127 (10) (2001) 1027-1032.

[18] M. Santasusana, J. Irazábal, E. Oñate, J. M. Carbonell, Ther7 Double Hierarchy Method. A parallel 3d contact method for 777 the interaction of spherical particles with rigid FE boundaries778 using the DEM, Comp Part Mech 3 (3) (2016) 407-428.

$19]$ N. Chakraborty, J. Peng, S. Akella, J. E. Mitchell, Proximity 780 Queries Between Convex Objects: An Interior Point Approach781 for Implicit Surfaces, IEEE T Robot 24 (1) (2008) 211-220. 782

[20] D. S. Lopes, M. T. Silva, J. A. Ambrósio, P. Flores, A mathe-783 matical framework for rigid contact detection between quadric784 and superquadric surfaces, Multibody Syst Dyn 24 (3) (2010)785 $255-280$.

[21] S. Ahmed, J. Harkness, L. Le Pen, W. Powrie, A. Zervos, Nu-787 merical modelling of railway ballast at the particle scale, Int J788 Numer Anal Met 40 (5) (2015) 713-737.

[22] A. Podlozhnyuk, C. Kloss, A contact detection method between 790 two convex super-quadric particles based on an Interior Point791 algorithm in the Discrete Element Method, in: IV International792 Conference on Particle-Based Methods, Barcelona, 2015. 793

[23] P. A. Cundall, Formulation of a three-dimensional distinct ele-794 ment model-Part I. A scheme to detect and represent contacts795 in a system composed of many polyhedral blocks, Int J Rock796 Mech Min 25 (3) (1988) 107-116.

[24] R. Hart, P. A. Cundall, J. Lemos, Formulation of a three-798 dimensional distinct element model-Part II. Mechanical cal-799 culations for motion and interaction of a system composed of 800 many polyhedral blocks, Int J Rock Mech Min 25 (3) (1988)801 $117-125$.

[25] E. G. Nezami, Y. M. A. Hashash, D. Zhao, J. Ghaboussi,803 Shortest link method for contact detection in discrete element804 method, Int J Numer Anal Meth Geomech 30 (8) (2006) 783-805 801.

[26] J. Eliáš, Simulation of railway ballast using crushable polyhe-807 dral particles, Powder Technol 264 (2014) 458-465.

27] F. Alonso-Marroquín, Spheropolygons: A new method tosog simulate conservative and dissipative interactions between $2 \mathrm{~d} 810$ complex-shaped rigid bodies, EPL 83 (1) (2008) 14001.

[28] F. Alonso-Marroquín, Y. Wang, An efficient algorithm for gran-812 ular dynamics simulations with complex-shaped objects, Granuls13 Matter 11 (5) (2009) 317-329.
[29] S. A. Galindo-Torres, D. M. Pedroso, Molecular dynamics simulations of complex-shaped particles using Voronoi-based spheropolyhedra, Phys Rev E 81 (6) (2010) 061303.

[30] V. Richefeu, G. Mollon, D. Daudon, P. Villard, Dissipative contacts and realistic block shapes for modeling rock avalanches, Eng Geol 149-150 (2012) 78-92.

[31] N. Ouhbi, C. Voivret, G. Perrin, J.-N. Roux, Railway Ballast: Grain Shape Characterization to Study its Influence on the Mechanical Behaviour, Procedia Engineering 143 (2016) 11201127.

[32] E. Oñate, J. Rojek, Combination of discrete element and finite element method for analysis of geomechanics problems, Comput Meth Appl M 193 (2004) 3087-3128.

[33] G. Casas, D. Mukherjee, M. A. Celigueta, T. I. Zohdi, E. Onate, A modular, partitioned, discrete element framework for industrial grain distribution systems with rotating machinery, Comp Part Mech (2015) 1-18.

[34] E. Oñate, F. Zárate, J. Miquel, M. Santasusana, M. A. Celigueta, F. Arrufat, R. Gandikota, K. Valiullin, L. Ring, A local constitutive model for the discrete element method. application to geomaterials and concrete, Comp Part Mech 2 (2) (2015) 139-160.

[35] O. Zienkiewicz, R. Taylor, D. Fox, The Finite Element Method for Solid and Structural Mechanics, Seventh Edition, Butterworth-Heinemann, Oxford, 2014.

[36] J. Ai, J.-F. Chen, J. M. Rotter, J. Y. Ooi, Assessment of rolling resistance models in discrete element simulations, Powder Technol 206 (3) (2011) 269-282.

[37] Y. C. Zhou, B. D. Wright, R. Y. Yang, B. H. Xu, A. B. Yu, Rolling friction in the dynamic simulation of sandpile formation, Physica A: Statistical Mechanics and its Applications 269 (2-4) (1999) 536-553.

[38] K. Iwashita, M. Oda, Rolling Resistance at Contacts in Simulation of Shear Band Development by DEM, J Eng Mech-ASCE 124 (3) (1998) 285-292.

[39] H. Sakaguchi, E. Ozaki, T. Igarashi, Plugging of the Flow of Granular Materials during the Discharge from a Silo, Int J Mod Phys B 07 (09n10) (1993) 1949-1963.

[40] A. Tasora, M. Anitescu, A complementarity-based rolling friction model for rigid contacts, Meccanica 48 (7) (2013) 16431659 .

[41] P. Dadvand, R. Rossi, E. Oñate, An object-oriented environment for developing finite element codes for multi-disciplinary applications, Arch Comput Method E 17 (3) (2010) 253-297.

[42] E. Tutumluer, Y. Qian, Y. M. A. Hashash, J. Ghaboussi, D. D. Davis, Discrete element modelling of ballasted track deformation behaviour, International Journal of Rail Transportation 1 (1-2) (2013) 57-73.

[43] C. Chen, B. Indraratna, G. McDowell, C. Rujikiatkamjorn, Discrete element modelling of lateral displacement of a granular assembly under cyclic loading, Comput Geotech 69 (2015) 474484.

[44] M. Melis Maynar, Embankments and Ballast in High Speed Rail, Revista de Obras Publicas 153 (2006) 7-26.

[45] Aggregates for railway ballast; EN 13450:2015.

[46] I. Farmer, Engineering Properties of Rocks, Spon, 1968.

[47] A. M. Howatson, P. G. Lund, J. D. Todd, Engineering tables and data, Chapman and Hall, London, U.K., 1972.

[48] A. Aikawa, Dynamic characterisation of a ballast layer subject to traffic impact loads using three-dimensional sensing stones and a special sensing sleeper, Constr Build Mater 92 (2015) 23-30.

[49] J. Harkness, A. Zervos, L. L. Pen, S. Aingaran, W. Powrie, Discrete element simulation of railway ballast: modelling cell pressure effects in triaxial tests, Granular Matter 18 (3) (2016) 65.

[50] D. W. Taylor, Fundamentals of soil mechanics, J. Wiley, 1948.

[51] E. Kabo, A numerical study of the lateral ballast resistance in railway tracks, Proceedings of the Institution of Mechanical Engineers, Part F: Journal of Rail and Rapid Transit 220 (4) (2006) 425-433. 
[52] J. Gallego, D. Gómez-Rey, A finite element solution for the lateral track buckling problem, Technical Report, TIFSA-RENFE Group (2001).

[53] J. v. t. Zand, J. Moraal, Ballast Resistance under Three Dimensional Loading, Tech. rep., Delft University of Technology (1997).

[54] V. D. H. Tran, M. A. Meguid, L. E. Chouinard, Discrete Element and Experimental Investigations of the Earth Pressure Distribution on Cylindrical Shafts, Int J Geomech 14 (1) (2014) 80-91. 